\title{
Kinetics of Ga droplet decay on thin carbon films
}

S Kodambaka, C Ngo, Justinas Palisaitis, P H. Mayrhofer, Lars Hultman and Per O A Persson

\section{Linköping University Post Print}

\section{Tweet}

N.B.: When citing this work, cite the original article.

Original Publication:

S Kodambaka, C Ngo, Justinas Palisaitis, P H. Mayrhofer, Lars Hultman and Per O A Persson, Kinetics of Ga droplet decay on thin carbon films, 2013, Applied Physics Letters, (102), 16, .

http://dx.doi.org/10.1063/1.4802758

Copyright: American Institute of Physics (AIP) http://www.aip.org/

Postprint available at: Linköping University Electronic Press

http://urn.kb.se/resolve?urn=urn:nbn:se:liu:diva-93860 


\title{
Kinetics of Ga droplet decay on thin carbon films
}

\author{
S. Kodambaka, ${ }^{1, a)}$ C. Ngo, ${ }^{1}$ J. Palisaitis, ${ }^{2}$ P. H. Mayrhofer,${ }^{3}$ L. Hultman, ${ }^{2}$ \\ and P. O. A. Persson ${ }^{2}$ \\ ${ }^{1}$ Department of Materials Science and Engineering, University of California Los Angeles, \\ Los Angeles, California 90095, USA \\ ${ }^{2}$ Department of Physics, Chemistry, and Biology (IFM), Linköping University, S-581 83 Linköping, Sweden \\ ${ }^{3}$ Institute of Materials Science and Technology, Vienna University of Technology, A-1040 Vienna, Austria
}

(Received 11 January 2013; accepted 9 April 2013; published online 22 April 2013)

\begin{abstract}
Using in situ transmission electron microscopy, we investigated the kinetics of liquid Ga droplet decay on thin amorphous carbon films during annealing at $773 \mathrm{~K}$. The transmission electron microscopy images reveal that liquid Ga forms spherical droplets and undergo coarsening/decay with increasing time. We find that the droplet volumes change non-linearly with time and the volume decay rates depend on their local environment. By comparing the late-stage decay behavior of the droplets with the classical mean-field theory model for Ostwald ripening, we determine that the decay of Ga droplets occurs in the surface diffusion limited regime. (C) 2013 AIP Publishing LLC [http://dx.doi.org/10.1063/1.4802758]
\end{abstract}

The phenomenon of Ostwald ripening refers to coarsening of large clusters at the expense of decaying smaller clusters as a means to minimize the total surface free energy. The cluster coarsening and decay process is governed by the GibbsThomson relation, according to which the equilibrium vapor pressure associated with a cluster increases exponentially with increasing curvature. The essential steps involved during coarsening and decay are: (1) attachment/detachment (also referred to as evaporation/condensation) of atoms at the cluster edges and (2) diffusion of atoms between the clusters. Classical mean-field theory models of Ostwald ripening, first developed by Lifshitz and Slyozov and later extended by others, ${ }^{1,2}$ have been useful in the identification of the mass transport mechanisms controlling the experimentally observed ripening of liquid droplets, as well as solid clusters on surfaces. ${ }^{3,4}$ For example, time-dependent changes in the radius $r(t)$ of a three-dimensional (3D) cluster are predicted to follow simple relationships of the form $r \propto t^{n}$, with the scaling exponents $n=1 / 3$ and $1 / 4$ corresponding to attachment/detachment- and diffusion-limited kinetics, respectively. Similar approach was later extended to analyze decay rates of individual two-dimensional islands on surfaces, from which ratelimiting steps and associated energetics controlling Ostwald ripening have been determined. ${ }^{5-8}$ While there is considerable literature on the kinetics of surface diffusion and coarsening of a variety of solids (elemental metals, ${ }^{7,9}$ semiconductors, ${ }^{6}$ and refractory compounds $\left.{ }^{10,11}\right)$, relatively fewer studies exist on the kinetics of coarsening and decay of liquid metals. ${ }^{3}$

Liquid metals such as gallium and related alloys are attractive for applications in high-temperature thermometry, in mirrors, as dental fillings in medicine, as coolants in microelectronics and nuclear reactors, and as alloying additives in metallurgy owing to a low vapor pressure, excellent wettability as well as good thermal and electrical conductivities. ${ }^{12}$ More recently, liquid metals are found to be promising for applications in plasmonics, ${ }^{13,14}$ as liquid-metal electrodes in

\footnotetext{
${ }^{\text {a) }}$ Author to whom correspondence should be addressed. Electronic mail: kodambaka@ucla.edu
}

batteries, ${ }^{15}$ as ultra-stretchable conductive wires, ${ }^{16}$ and as catalysts for the growth of nanowires ${ }^{17,18}$ and graphene. ${ }^{19,20}$ For any of these applications, thermochemical stability of liquidmetal/solid interfaces is important since it affects properties such as wettability, adhesion, chemical reactivity, reflectivity, thermal and electrical conductivities, optoelectronic properties, and the size of the liquid droplets. Here, we focus on understanding the thermal stability of liquid-gallium/amorphous-carbon interfaces. While carbon is expected to be insoluble in liquid gallium, previous studies have suggested otherwise and due to the large difference in the surface energies, liquid gallium does not wet amorphous carbon and forms droplets with wetting angles exceeding $120^{\circ}{ }^{19,21-23}$

In this letter, we present in situ transmission electron microscopy (TEM) studies of the decay behavior of Ga droplets on amorphous carbon thin films during annealing. From the time-lapsed TEM images, we find that smaller droplets shrink and the volume decay rates depend on the local environment, characteristic of surface-diffusion-limited Ostwald ripening. We confirm this behavior by comparing the latestage decay behavior of the droplets with classical meanfield theory model for Ostwald ripening.

All of our experiments were carried out on amorphous carbon thin films deposited using $\mathrm{Ga}^{+}$ions in a FEI Nova 600 Nanolab DualBeam focused-ion-beam (FIB) system equipped with a scanning electron microscope and facilities for electron- and ion-beam induced deposition of Pt, W, and C. Previous studies have shown that FIB deposition of thin films using $\mathrm{Ga}^{+}$ions leads to implantation of $\mathrm{Ga}$, which upon heating forms liquid droplets on the surfaces of the deposited layers. ${ }^{21,24-28}$ In this report, we employ a similar approach to prepare Ga droplets on amorphous carbon thin films as described below.

We used $\mathrm{ZrB}_{2} / \mathrm{Al}_{2} \mathrm{O}_{3}(0001)$ thin film samples as the substrate and prepared electron-transparent cross-sectional TEM (XTEM) samples via FIB milling using $30 \mathrm{kV} \mathrm{Ga}^{+}$ions. Prior to milling, the $\mathrm{ZrB}_{2} / \mathrm{Al}_{2} \mathrm{O}_{3}(0001)$ thin film surface was protected by a $1.5 \mu \mathrm{m}$ thick layer of carbon, deposited initially using $5 \mathrm{kV}$ and $6.3 \mathrm{nA}$ electron beams and afterwards using 


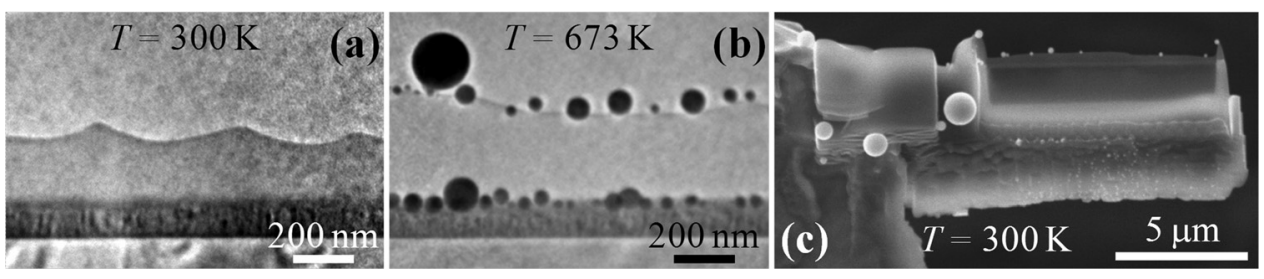

FIG. 1. (a) Representative bright-field XTEM image acquired at room temperature from $\mathrm{ZrB}_{2}-\mathrm{Al}_{2} \mathrm{O}_{3}(0001)$ interface prepared via FIB milling. (b) XTEM image of the same sample obtained during annealing at $T=673 \mathrm{~K}$. In the images, the $\mathrm{Al}_{2} \mathrm{O}_{3}(0001)$ substrate is at the bottom. The upper layers with dark and light grey contrasts are $\mathrm{ZrB}_{2}$ and amorphous carbon, respectively. In Fig. 1(b), liquid Ga droplets appear as nearly spherical objects on top of the carbon film. (c) Scanning electron microscopy image acquired from another FIB-cut $\mathrm{ZrB}_{2} / \mathrm{Al}_{2} \mathrm{O}_{3}(0001)$ thin film sample after annealing. In this experiment, the sample was gradually heated to $T=773 \mathrm{~K}$ over a period of $23 \mathrm{~min}$ and held at that $T$ for an additional $15 \mathrm{~min}$ before cooling to room temperature. The brighter contrast spherical objects are Ga droplets that appear all over the sample with large droplets predominantly located on the sides of the sample and several smaller droplets on the thicker, back end of the sample.

$30 \mathrm{kV}$ and $0.3 \mathrm{nA}$ ion beams. The FIB-milled samples were attached to the TEM grid by depositing Pt via FIB. Final thinning to electron transparency was carried out by $10 \mathrm{kV}$ ion beams. Fig. 1(a) is a representative bright-field XTEM image of the as-prepared sample. In the image, the darker contrast layer is the $\mathrm{ZrB}_{2}$ film supported by $\mathrm{Al}_{2} \mathrm{O}_{3}(0001)$ at the bottom and covered by an amorphous carbon layer. In this projection view, an $\sim 250 \mathrm{~nm}$ thick carbon layer is visible on the top surface of the sample. Upon heating, we observe multiple, nearly spherical liquid droplets along the edges of the carbon film, as shown in Fig. 1(b). Selected area electron diffraction patterns acquired from the droplets and the carbon layers indicate that they are amorphous and remain so during the course of our heating experiments. Energy dispersive spectroscopy (EDS) measurements acquired from the XTEM sample, both before and after annealing, indicate that a significant amount of $\mathrm{Ga}$ was present within the carbon film prior to heating and in the droplets observed post-annealing after air-exposure. EDS data also indicated a significant fraction of oxygen but this is likely due to air exposure and not expected in the droplets since gallium oxide does not melt at $773 \mathrm{~K}$. The presence of carbon, if any, ${ }_{19,21-23}$ within the droplets could not be determined accurately from EDS due to the large background signal of carbon from the surrounding region. This procedure of forming $\mathrm{Ga}$ droplets is highly reproducible and we have obtained similar results, i.e., Ga droplets on carbon films on FIB-prepared XTEM samples of $\mathrm{C} / \mathrm{Ti} / \mathrm{Si}, \mathrm{Zr} / \mathrm{Al}_{2} \mathrm{O}_{3}(0001)$, and bare $\mathrm{Al}_{2} \mathrm{O}_{3}(0001)$ substrates. The size and areal coverage of these Ga droplets, however, vary with the heating rate and the substrate temperature.

In situ annealing experiments were carried out using a Gatan double-tilt heating holder in a $200 \mathrm{kV}, \mathrm{LaB}_{6}$, Philips CM20ST TEM (base pressure $\sim 10^{-7}$ Torr). The sample was air-transferred into the TEM and held at ambient temperature until the base pressure recovered. Then, the sample was heated to $T=773 \mathrm{~K}$ in intervals of $100 \mathrm{~K}$ and holding at each temperature for approximately $20 \mathrm{~min}$. TEM images were acquired in bright-field mode at regular intervals ( $\sim 20-60$ s). Image magnification and acquisition times were varied to check for the influence of electron beam irradiation on the droplet dynamics. We do not observe any such effects on the results presented here. The droplet radii were determined from the TEM images using the IMAGEJ, an image processing software.

Figure 2 shows a series of TEM images acquired as a function of time $t$ during annealing the sample at $T=773 \mathrm{~K}$. The near-spherical morphology of the droplets and the minimal contact with the surface (see Figs. 1(b) and 2) suggest that Ga does not wet carbon. This is plausible since molten $\mathrm{Ga}$ has significantly higher surface energy $\left(\gamma \approx 4 \mathrm{eV} / \mathrm{nm}^{2} \text { at } 773 \mathrm{~K}\right)^{29,30}$ than that of amorphous carbon $\left(\gamma \approx 0.25 \mathrm{eV} / \mathrm{nm}^{2}\right) .{ }^{31} \mathrm{We}$ find that, as time progresses, most of the droplets decrease in size and eventually disappear. The large droplet labeled $A$ and several other large droplets outside of the field of view, which are typically found at the ends of the sample (see Fig. 1(c)), coarsen and remain on the surface. In contrast with previous in situ TEM observations, we do not observe graphitic shell formation around the droplets. ${ }^{19,21,22,24} \mathrm{We}$ do, however, observe small remnants that do not change in size after the bulk of the droplets dissolve. While the images lack sufficient resolution to identify these features, presumably they are graphitic shells as has been reported in the literature.

We now focus on the droplet decay behavior. In the case of material evaporation from the surface into vacuum and/or diffusion into the carbon layer, sizes of all of the droplets

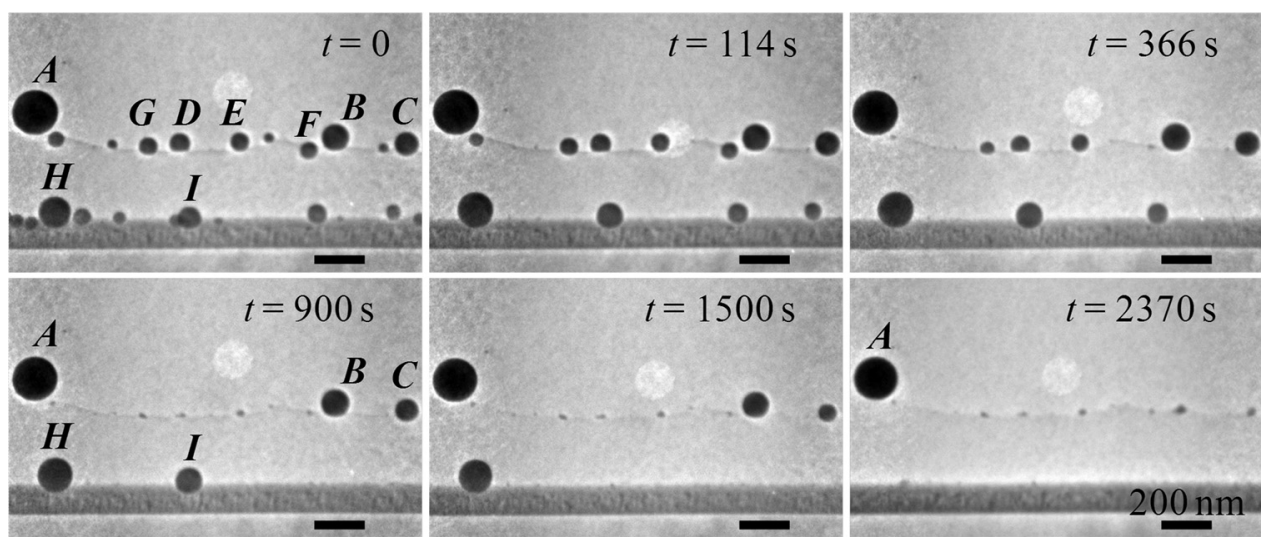

FIG. 2. Typical bright-field XTEM images acquired from the sample shown in Fig. 1 as a function of time $t$ during annealing at $T=773 \mathrm{~K}$. The lighter grey contrast circular feature visible near the center of all the images is an artifact of the camera. 
should decrease with time. Clearly, not all droplets decay in our experiments. Moreover, the vapor pressure of $\mathrm{Ga}$ at $T=773 \mathrm{~K}$ is $<10^{-9}$ Torr $^{32}$ and the solubility of Ga in carbon is negligible. Hence, we rule out both of these processes (evaporation and bulk diffusion) as contributing factors to the observed droplet decay. Instead, we suggest (and justify below) that the observed disappearance of droplets is a consequence of Ostwald ripening. ${ }^{1,3}$

In order to validate our hypothesis, we measured timedependent changes in droplet sizes. Figure 3(a) shows plots of droplet radii $r$ vs. $t$ data for the 9 droplets labeled $A-I$ in Fig. 2. In case the observed decay of Ga droplets is due to diffusion of $\mathrm{Ga}$ into the bulk of the sample or evaporation into vacuum, all of the droplets would decrease in size. ${ }^{33-36} \mathrm{We}$ find that droplets $B-I$ exhibit decay while the size of droplet $A$ remains nearly constant at all $t$. Moreover, the rates of changes in droplet size are not the same for all the droplets. For example, the sizes of smaller droplets $F$ and $G$ decrease continuously, while the sizes of larger droplets $B$ and $C$ change little initially and decrease at later times. The observed variations in decay rates are similar to those reported for Ostwald ripening of 2D islands on surfaces and are attributed to local variations in adatom concentrations (equivalent to $2 \mathrm{D}$ vapor pressure) around the islands. ${ }^{6,7,11}$

In order to compare our experimental observations with the scaling relations of the form $r \propto t^{n}$ predicted by classical mean-field theory models, we focus on the late-stage decay behavior of the droplets. This is because the scaling relations are derived under two key assumptions: (i) the surface adatom concentration $C_{s}$ is uniform and equal to $C_{\infty}$, the equilibrium concentration associated with a planar surface $(r=\infty)$ and (ii) the adatom concentration $C_{r}$ associated with a droplet of radius $r$ is given by the linear expansion of the Gibbs-Thomson relation as $C_{r} \approx C_{\infty}\left(1+2 \gamma \Omega / r \mathrm{k}_{\mathrm{B}} T\right)$, where $\Omega=0.02 \mathrm{~nm}^{3}$ is the atomic volume of $\mathrm{Ga}$ in liquid phase. While $C_{s}$ will always be higher than $C_{\infty}$ for non-zero coverage of droplets on the surface, the droplet decay rate $\mathrm{d} r / \mathrm{d} t$ is proportional to $\left(C_{r}-C_{s}\right)$, which is $\approx\left(C_{r}-C_{\infty}\right)$ for low coverages and small droplets. Fig. 3(b) shows representative log$\log$ plots of $r$ vs. $\left(t_{\text {end }}-t\right)$ data of droplets $B-E$. In the plots, only the late stages of decay are shown and $t_{\text {end }}$ is the time at which the droplet has completely disappeared as determined from the TEM images and is accurate to within $5 \mathrm{~s}$. From the linear least-squares fits to the data, we obtain $n$ values of $0.25 \pm 0.01,0.22 \pm 0.01,0.22 \pm 0.01$, and $0.27 \pm 0.02$ for $B$,
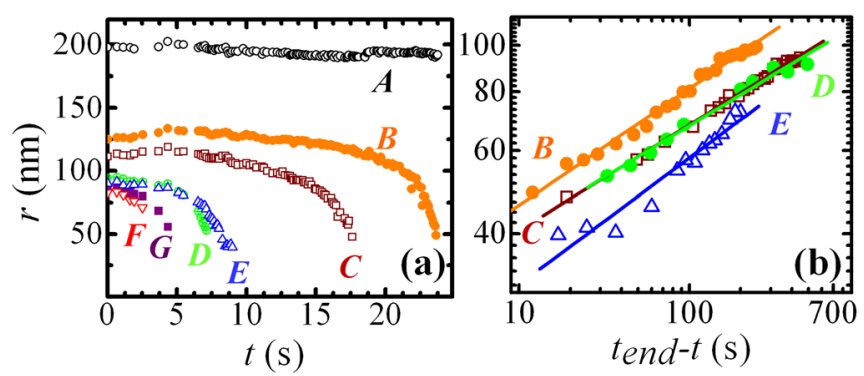

FIG. 3. (a) Droplet radii ( $r$ ) plotted as a function of annealing time $t$. The labels $A-G$ correspond to the droplets in Fig. 2. (b) Log-log plots of $r$ vs. $\left(t_{\text {end }}-t\right)$ for droplets $B-E . t_{\text {end }}$ is defined as the time at which the droplet has completely disappeared. The solid lines are linear least-squares fits to the data, whose slopes yield the scaling exponents $n$.
$C, D$, and $E$, respectively. This value is closer to the meanfield theory predicted value of $n=1 / 4$ for coarsening/decay in the diffusion-limited regime rather than the value of $n=1 / 3$ expected for attachment-limited decay kinetics. We note that exponents smaller than the predicted values can be observed because the scaling relations are derived based upon meanfield theory approximations. ${ }^{5,7,11}$ Most significant deviations from the predicted values arise when the condition $C_{r} \approx$ $C_{\infty}\left(1+2 \gamma \Omega / r \mathrm{k}_{\mathrm{B}} T\right)$ is not satisfied, i.e., when the droplets are small such that $2 \gamma \Omega / r \mathrm{k}_{\mathrm{B}} T \gg 0$. Substituting for $\Omega, \gamma$, and $\mathrm{T}$, we find that the linear expansion is accurate to within $1 \%$ for droplet sizes $r \geq 20 \mathrm{~nm}$. In our experiments, all of the droplets are larger than $20 \mathrm{~nm}$ (see Fig. 3(b)) and hence the mean-field theory predictions apply. Therefore, we suggest that surface diffusion is the rate-limiting process controlling the observed decay of $\mathrm{Ga}$ droplets. In the surface diffusion limited regime, assuming spherical droplets, $\frac{d r}{d t} \approx\left(\frac{D C_{\infty} \gamma \Omega^{2}}{\mathrm{k}_{\mathrm{B}} T}\right)\left(\frac{1}{r^{3}}\right)$, where $D$ is surface diffusivity of $\mathrm{Ga}$ on amorphous carbon layers. ${ }^{2,37}$ From the linear fits in Fig. 3(b), we obtain $D C_{\infty}$ values between $\sim 4 \times 10^{5}$ and $\sim 2 \times 10^{7} \mathrm{~s}^{-1}$. Assuming a prefactor of $10^{12} \mathrm{~s}^{-1}$, the activation energies associated with these $D C_{\infty}$ values are between 0.7 and $1.0 \mathrm{eV}$, reasonable for surface mass transport of metals. ${ }^{7}$

In summary, we investigated the decay behavior of liquid gallium droplets on top of amorphous carbon layers using in situ transmission electron microscopy. We find that the droplets undergo Ostwald ripening via diffusion of $\mathrm{Ga}$ along the carbon thin film surface. Our results, which help understand the factors controlling the thermal stability of Ga droplets on carbon surfaces, may be useful in the fabrication of size-controlled $\mathrm{Ga}$ droplets for plasmonics and other applications.

We gratefully acknowledge support from the AFOSR (Dr. Ali Sayir) FA9550-10-1-0496, STINT, the Swedish Foundation for International Cooperation in Research and Higher Education, The Swedish Research Council, the Austrian Science Fund FWF, START project Y371, and The Knut and Alice Wallenberg Foundation for the Ultra Electron Laboratory at Linköping. We thank Mr. Noah Bodzin and the Nanoelectronics Research Facility in the UCLA Henry Samueli School of Engineering for assistance with focused ion beam milling.

${ }^{1}$ I. M. Lifshitz and V. V. Slyozov, J. Phys. Chem. Solids 19(1), 35-50 (1961).

${ }^{2}$ P. Wynblatt and N. Gjostein, Acta Metall. 24(12), 1165-1174 (1976).

${ }^{3}$ M. Zinke-Allmang, L. C. Feldman, and M. H. Grabow, Surf. Sci. Rep. 16(8), 377-463 (1992).

${ }^{4}$ J. Viñals and W. Mullins, J. Appl. Phys. 83(2), 621-628 (1998).

${ }^{5}$ K. Morgenstern, G. Rosenfeld, and G. Comsa, Phys. Rev. Lett. 76(12), 2113-2116 (1996).

${ }^{6}$ N. Bartelt, W. Theis, and R. Tromp, Phys. Rev. B 54(16), 11741 (1996).

${ }^{7}$ M. Giesen, Prog. Surf. Sci. 68(1), 1-154 (2001).

${ }^{8}$ S. Kodambaka, S. V. Khare, I. Petrov, and J. E. Greene, Surf. Sci. Rep. 60(5), 55-77 (2006).

${ }^{9}$ W. W. Pai, A. K. Swan, Z. Zhang, and J. Wendelken, Phys. Rev. Lett. 79(17), 3210-3213 (1997).

${ }^{10}$ S. Kodambaka, V. Petrova, S. V. Khare, D. Gall, A. Rockett, I. Petrov, and J. E. Greene, Phys. Rev. Lett. 89(17), 176102 (2002).

${ }^{11}$ S. Kodambaka, V. Petrova, A. Vailionis, I. Petrov, and J. E. Greene, Surf. Sci. 526(1-2), 85-96 (2003).

${ }^{12}$ V. Y. Prokhorenko, V. V. Roshchupkin, M. A. Pokrasin, S. V. Prokhorenko, and V. V. Kotov, High Temp. 38(6), 954-968 (2000). 
${ }^{13}$ S. R. C. Vivekchand, C. J. Engel, S. M. Lubin, M. G. Blaber, W. Zhou, J. Y. Suh, G. C. Schatz, and T. W. Odom, Nano Lett. 12(8), 4324-4328 (2012).

${ }^{14}$ C. W. Yi, T. H. Kim, W. Y. Jiao, Y. Yang, A. Lazarides, K. Hingerl, G. Bruno, A. Brown, and M. Losurdo, Small 8(17), 2721-2730 (2012).

${ }^{15}$ R. D. Deshpande, J. C. Li, Y. T. Cheng, and M. W. Verbrugge, J. Electrochem. Soc. 158(8), A845-A849 (2011).

${ }^{16}$ S. Zhu, J.-H. So, R. Mays, S. Desai, W. R. Barnes, B. Pourdeyhimi, and M. D. Dickey, "Ultrastretchable Fibers with Metallic Conductivity Using a Liquid Metal Alloy Core," Adv. Funct. Mater. (published online).

${ }^{17}$ K. A. Dick, Prog. Cryst. Growth Charact. Mater. 54(3), 138-173 (2008).

${ }^{18}$ H. L. Zhou, M. Pozuelo, R. F. Hicks, and S. Kodambaka, J. Cryst. Growth 319(1), 25-30 (2011).

${ }^{19}$ J. Fujita, R. Ueki, Y. Miyazawa, and T. Ichihashi, J. Vac. Sci. Technol. B 27(6), 3063-3066 (2009).

${ }^{20}$ H. Hiura, M. V. Lee, A. V. Tyurnina, and K. Tsukagoshi, Carbon 50(14), 5076-5084 (2012).

${ }^{21}$ R. Kometani, T. Ichihashi, S. Warisawa, and S. Ishihara, Jpn. J. Appl. Phys., Part 1 48(6), 06FE01 (2009).

${ }^{22}$ R. Ueki, T. Nishijima, T. Hikata, S. Ookubo, R. Utsunomiya, T. Matsuba, and J. Fujita, Jpn. J. Appl. Phys., Part 1 51(6), 06FD28 (2012).

${ }^{23}$ C. Y. Wang, Y. C. Chen, W. H. Chu, C. P. Liu, and C. B. Boothroyd, J. Mater. Res. 24(4), 1388-1394 (2009).
${ }^{24}$ J. Fujita, T. Ichihashi, S. Nakazawa, S. Okada, M. Ishida, Y. Ochiai, T. Kaito, and S. Matsui, Appl. Phys. Lett. 88(8), 083109 (2006).

${ }^{25}$ W. J. MoberlyChan, D. P. Adams, M. J. Aziz, G. Hobler, and T. Schenkel, MRS Bull. 32(5), 424-432 (2007).

${ }^{26}$ Q. Hu and W. O'Neill, Appl. Surf. Sci. 256(20), 5952-5956 (2010).

${ }^{27}$ C. C. Lee, G. Proust, G. Alici, G. M. Spinks, and J. M. Cairney, J. Microsc. 248(2), 129-139 (2012).

${ }^{28}$ P. Peter, B. Lothar, and S. Bernd, Nanotechnology 23(47), 475304 (2012).

${ }^{29}$ B. Keene, Int. Mater. Rev. 38(4), 157-192 (1993).

${ }^{30}$ B. Alchagirov and A. Mozgovoi, High Temp. 43(5), 791-792 (2005).

${ }^{31}$ J. S. Chen, S. P. Lau, B. K. Tay, G. Y. Chen, Z. Sun, Y. Y. Tan, G. Tan, and J. W. Chai, J. Appl. Phys. 89(12), 7814-7819 (2001).

${ }^{32}$ D. R. Lide, CRC Handbook of Chemistry and Physics (CRC Press, 2012).

${ }^{33}$ K. F. McCarty, J. A. Nobel, and N. C. Bartelt, Nature 412(6847), 622-625 (2001).

${ }^{34}$ S. Kodambaka, S. V. Khare, W. Swiech, K. Ohmori, I. Petrov, and J. E. Greene, Nature 429(6987), 49-52 (2004).

${ }^{35}$ S. Kodambaka, N. Israeli, J. Bareno, W. Swiech, K. Ohmori, I. Petrov, and J. E. Greene, Surf. Sci. 560(1-3), 53-62 (2004).

${ }^{36}$ S. J. Tang, S. Kodambaka, W. Swiech, I. Petrov, C. P. Flynn, and T. C. Chiang, Phys. Rev. Lett. 96(12), 126106 (2006).

${ }^{37}$ In this relation, we assume that the droplets are spherical and ignore the logarithmic diffusional screening term that arises as part of the solution to 2D Laplace equation. 